\title{
Uma construção do Perfil Epistemológico de licenciandos em Física acerca da dualidade onda-partícula em Mecânica Quântica, após o uso de bancadas virtuais: um estudo a partir do discurso gestual e verbal
}

\author{
Robson Trevisan - PPGECIM/ULBRA. robsontrevi@gmail.com \\ Agostinho Serrano de Andrade Neto - PPGECIM/ULBRA. asandraden@gmail.com
}

Resumo: O presente artigo busca apresentar o esboço do perfil epistemológico acerca da dualidade onda-partícula de estudantes do sétimo período do curso de Licenciatura em Física, após a utilização de ferramentas hiperculturais sob a forma de bancadas virtuais. A condução desta investigação converge para a tentativa de relacionar as imagens mentais e drivers dos alunos com as principais interpretações da Mecânica Quântica, a ponto de mapear as filosofias com maior destaque e influência no espírito do sujeito na construção de conhecimentos acerca da dualidade. O referencial teórico adotado para leitura dos dados foi o da ideia de perfil epistemológico Bachelardiano, com a intenção de abarcar a postura epistemológica expressada pelos alunos. Também utilizamos a Teoria da Mediação Cognitiva, procurando explicar como a mediação com o computador afeta a estrutura cognitiva dos estudantes. Os resultados foram obtidos após as análises realizadas sobre os pré-testes, pós-testes e gestos descritivos obtidos das imagens de vídeo, gravadas durante as entrevistas do pós-teste, sendo que essas entrevistas foram conduzidas seguindo o método "Report Aloud". O perfil epistemológico dos alunos foi traçado de acordo com as interpretações privadas, inferidas a partir dos drivers conceituais, manifestados e identificados, e do conteúdo do discurso dos mesmos.

Palavras-chave: Bancadas Virtuais; Mecânica Quântica; Teoria da Mediação Cognitiva; Perfil Epistemológico; Report Aloud.

\section{A construction Epistemological Profile of undergraduate in physics about the wave-particle duality in quantum mechanics, after the use of virtual stands: a study from the gestural and verbal speech}

\begin{abstract}
The present paper seeks to present the epistemological profile sketch on the wave-particle duality, students of the seventh period of the Bachelor's Degree in Physics, after using hiperculturais tools in the form of virtual stands. Conducting this research converges to attempt to relate the mental images and drivers of the students with the main interpretations of quantum mechanics, as to map the philosophies more prominence and influence in the spirit of the subject in the construction of knowledge about duality. The theoretical framework adopted for reading the data was the idea of epistemological profile Bachelardian with the intention of embracing the epistemological stance expressed by the students. We also use the Theory of Cognitive Mediation, seeking to explain how mediation with the computer affects the cognitive structure of students. The results were obtained after the analysis performed on the pre test, post-test and descriptive gestures obtained video images, recorded during interviews post-test, and these interviews were conducted following the "Report Aloud". The epistemological profile of students was drawn according to private interpretation, inferred from the conceptual, expressed and identified drivers and speech contents.
\end{abstract}

Keywords: Virtual Work Benches, Epistemological Profile, Quantum Mechanics; Cognitive Mediation Networks Theory, Report Aloud.

V. $14 \mathrm{~N}^{\circ} 1$, julho, 2016 


\section{Introdução}

A Teoria Quântica é tida como uma teoria sólida e de sucesso, pois suas previsões têm sido comprovadas experimentalmente ao longo deste século com grande precisão. Mesmo diante desse cenário, "[...] é notável, no entanto, que a mecânica quântica tenha dezenas de interpretações diferentes. Ou seja, mesmo havendo concordância sobre o formalismo da teoria, não há consenso sobre o que ela diz em relação à realidade" (Pessoa Jr., 2008, p. 32).

O extenso reconhecimento da expressão da Mecânica Quântica (MQ) em tecnologias atuais e seu papel na formação científica e na cultura da ciência, até mesmo pela crescente apropriação da MQ para misticismos de toda a espécie, disseminando informações oriundas de uma pseudociência por meio de uma "pressão social", têm acentuado a investigação de vastas formas de apresentar $o$ assunto em cursos introdutórios de Física Moderna, em diversos níveis de ensino. "As dificuldades interpretativas dessa teoria dizem respeito tanto à forma pela qual a teoria se relaciona com os fenômenos quanto ao delineamento de uma ontologia que lhe seja apropriada" (Ostermann e Prado, 2005, p. 194). Nos cursos em que a MQ é apresentada, o ensino tradicional ainda se encontra enraizado, dando ênfase aos algoritmos de resoluções das equações matemáticas. De acordo com Greca, Moreira e Herscovitz (2001), as abordagens típicas desses cursos não criam condições para que os alunos aprendam uma nova forma de interpretar os fenômenos da MQ.

Montenegro e Pessoa Jr (2002) apontam em uma das conclusões de seu trabalho que o experimento da dupla fenda para elétrons individuais é o mais paradigmático e importante da MQ, e que, apesar disso, aproximadamente metade dos alunos respondeu erroneamente as questões propostas que envolviam tal ponto. Esses resultados, segundo os autores, devem-se ao fato de os cursos de MQ serem excessivamente voltados para os cálculos, negligenciando os conceitos e as questões interpretativas.

Schuck e Serrano (2004) utilizam bancadas virtuais para o ensino de diversos conceitos da MQ na disciplina de Estrutura da Matéria, em um curso de Química em nível superior, e observam que os estudantes têm dificuldade em explicar o comportamento dual dos objetos quânticos, possivelmente, segundo os autores, devido à utilização da noção partícula como obstáculo epistemológico. Essa dificuldade só é superada pela dificuldade ainda maior de explicar o comportamento dual de objetos quânticos virtuais. Resultados semelhantes são observados por Trevisan e Andrade Neto (2014).

Após a identificação da importância do tema da dualidade onda-partícula no ensino da MQ, mantendo a preocupação na abordagem conceitual, e não apenas nos processos de cálculos matemáticos, e também a crescente aparição das Tecnologias da Informação e Comunicação (TIC) voltadas aos processos educativos, proporcionando a utilização de softwares que simulam experimentos em regime quântico, esta pesquisa toma como proposta de trabalho o levantamento do perfil epistemológico, de licenciandos em Física, diante da noção de conceitos acerca da dualidade.

\section{A Teoria da Mediação Cognitiva (TMC)}

A TMC é uma teoria contextualista e construtivista, fundamentada e relacionada com diferentes escolas de pensamento, representadas pelos seguintes autores: Jean Piaget, Gérard Vergnaud, Lev Semenovich Vygotsky e Robert Sternberg. Um desafio importante para a teoria é o de "[...] fornecer uma síntese teórica coerente de teorias psicológicas e estruturais que são geralmente vistas como separadas, ou mesmo em conflito umas com as outras, de modo a produzir um modelo unificado" (Souza, et al., 
2012, p. 2). Considera-se uma aplicação importante da TMC a sua compreensão das mudanças individuais ou coletivas, associadas à introdução das tecnologias como ferramentas externas ao pensamento dos indivíduos.

A TMC parte do princípio de que cognição humana depende fundamentalmente do processamento de informações e que o cérebro humano isolado apresenta-se como insuficiente para explicar a maior parte do desempenho cognitivo, com os quais pode-se concluir que outros mecanismos de processamento de informação estão envolvidos. $\mathrm{O}$ autor constrói um conjunto de conceitos, dentro do seu proposto referencial teórico, dos quais nos chamam atenção os "mecanismos externos de mediação" e os "mecanismos internos de mediação", buscando trazer uma perspectiva diferenciada no que se refere a considerar a chamada cognição externa (ao cérebro). Pode-se, aqui, citar o fato de o uso de dispositivos eletrônicos - computadores, tablets e smartphones - se dar por um processo de mediação. Os mecanismos internos referem-se à estrutura mental que gerencia algoritmos, códigos e dados que permitem a conexão, interação e integração entre o processamento interno do cérebro e do processamento extracerebral feito pelas estruturas no ambiente, trabalhando tanto como um "driver de hardware" quanto como um "protocolo de rede"- (Souza, 2004).

À vista disso, de acordo com Souza (2004, p. 65), “[...] tais elementos [extracerebrais] só poderão efetivamente ser de utilidade para um indivíduo, se este dispuser de uma forma de interagir eficazmente com eles, segundo a necessidade e de modo adequado" com dispositivos existentes na estrutura intracerebral que permitam traduzir as entradas, as saídas e o processamento entre eles. Em resumo, são os drivers que possibilitam a mediação com estruturas do ambiente. Esses drivers são originados dos mecanismos de mediação externa utilizados pelo indivíduo, que podem ser de natureza psicofísica (quando o indivíduo utiliza de objetos do ambiente como mecanismo de processamento extracerebral), social (devido à conexão social com outros indivíduos por meio da linguagem), cultural (devido à utilização de ferramentas culturais, como livros, etc) ou hipercultural (utilização das TIC).

Diante deste cenário, utilizaremos a TMC como referencial teórico, em razão de sua abordagem relacionada à mediação hipercultural, em especial mediante o uso de computadores, sendo essas ferramentas tecnológicas responsáveis pela realização de processamentos externos de informação, interagindo com os mecanismos internos de processamento e, como consequência, modificando a estrutura cognitiva do indivíduo.

\section{A ideia de Perfil Epistemológico Bachelardiano}

O positivismo atribuído à pesquisa científica, bem como a objetividade no trabalho dos cientistas, é um fator que contraria as ideias de Bachelard, apresentadas em suas obras, como "O Novo Espírito Científico" (1985) e "A Filosofia do Não" (1991). Ressalta, então, a necessidade de construção de um pensamento complexo para a ciência e uma postura de reflexão filosófica sobre a prática científica.

Bachelard propõe executar diálogos ininterruptos entre valores experimentais e os valores racionais, característica da contemporaneidade, nos quais

[...] o empirismo e o racionalismo estão ligados, no pensamento científico, por um estranho laço tão forte como o que une o prazer à dor. Com efeito, um deles triunfa dando razão ao outro: o empirismo precisa ser compreendido; o racionalismo precisa ser aplicado. Um empirismo sem leis claras, sem leis coordenadas, sem leis dedutivas não pode ser nem pensado, nem ensinado; um racionalismo sem provas palpáveis, sem aplicação à realidade imediata não pode convencer plenamente. Prova-se o valor real de uma lei empírica 
fazendo dela a base de um raciocínio. Legitima-se um raciocínio fazendo dele a base de uma experiência (Bachelard, 1991, p. 4).

Com o intuito de mostrar que diferentes filosofias podem estar presentes na mesma acepção de um conceito, ainda que algumas delas sejam conscientemente consideradas inadequadas para caracterizar determinada noção do conhecimento científico, Bachelard (1991) apresenta ideias vinculadas ao termo por ele denominado como "perfil epistemológico", "[...] uma escala polêmica suficiente para localizar os diversos debates da filosofia da Ciência, para impedir a confusão dos argumentos" (Bachelard, 1991, p. 55). O perfil epistemológico “[...] deve sempre referir-se a um conceito designado, de ele apenas ser válido para um espírito particular que se examina num estádio particular da sua cultura" (Bachelard, 1991, p. 25). Percebe-se, então, que não se pode fixar um conceito a uma filosofia, mas, sim, numa diversidade de aspectos filosóficos que definem uma evolução temporal do conceito. De acordo com Bachelard (1991, p. 66), "Cada filosofia fornece apenas uma banda do espectro nocional, e é necessário agrupar todas as filosofias para termos o espectro nocional completo de um conhecimento particular".

Fazendo uso das ideias de Bachelard a respeito do perfil epistemológico do sujeito, pretende-se, neste trabalho, investigar as correntes filosóficas dos estudantes em relação ao conceito do comportamento dual da matéria e da radiação eletromagnética, após os mesmos realizarem atividades utilizando ferramentas hiperculturais.

\subsection{A relação entre as visões filosóficas e suas noções acerca da dualidade}

Bachelard (1991) relacionou as suas interpretações privadas acerca dos conceitos de massa e energia com as visões filosóficas pertinentes e responsáveis por conduzir determinadas compreensões. Através dessa ideia, pretende-se, aqui, traçar uma relação entre as noções construídas ao longo da história sobre a dualidade e as visões filosóficas que são responsáveis pela estruturação do perfil epistemológico do indivíduo. Esta correspondência é sugerida por Pinto e Zanetic (1999) em meio as suas investigações sobre a inserção da MQ no Ensino Médio, nas quais utilizam a ideia de Perfil Epistemológico como referencial filosófico. Desta forma, as filosofias que serão adotadas e vinculadas às noções acerca da natureza da luz estão descritas a seguir.

Realismo Ingênuo: Também pode ser denominado como Animismo, sendo considerado por Bachelard (1991) como um pensamento mais primitivo do indivíduo, o qual antecede o estágio científico da noção sobre determinados conceitos e fenômenos. A percepção de que a luz seria propriedade dos olhos e a concepção aristotélica de que a luz era resultado da atividade de um determinado meio, cuja vibração provocaria o movimento de humores presentes nos olhos integram-se na filosofia animista (Rocha, 2002).

Empirismo claro e positivista: Assume-se empirismo claro e positivista como a visão que trata as noções explicadas por meio de fatos empíricos, em cujas observações dos feixes de luz em determinadas situações são as principais investigações na construção do conceito sobre a natureza da luz. Por exemplo, a observação de feixes de luz ao atravessarem fendas de uma janela ou em qualquer outro objeto.

Racionalismo Clássico: Para a visão racional, é tomada como referência a categorização apresentada por Pinto e Zanetic (1999), na qual adotam para esta filosofia modelos com 
fundamentação teórico-matemática, a exemplo do modelo corpuscular de Newton e o ondulatório de Huygens.

Ultra-racionalismo: Optou-se por utilizar o ultra-racionalismo, sendo essa a visão filosófica que agrupa o racionalismo completo e racionalismo discursivo adotados por Bachelard (1991). Trata-se de um pensamento mais sofisticado, ou seja, é a evolução filosófica de um conhecimento científico particular, seguindo um caminho que se inicia no animismo e tem o ultra-racionalismo como ponto de chegada. Para o ultraracionalismo, é concebida toda a explicação que faz menção às interpretações modernas da MQ para a natureza da luz. Atribui-se, por exemplo, a visão ondulatória de Schrödinger, a corpuscular de Landé, a dualista realista de De Broglie e Bohm e a dualista positivista de Bohr.

\section{Problema de Pesquisa e Métodos}

O problema de pesquisa norteador deste trabalho circunda o seguinte questionamento: Quais são os aspectos dos perfis epistemológicos de estudantes do curso de licenciatura em Física acerca do tema da dualidade onda-partícula, após a mediação digital caracterizada pelo uso de laboratórios virtuais (ou bancadas virtuais)?

Portanto, o presente artigo tem como objetivo esboçar os perfis epistemológicos dos alunos por meio da investigação das imagens mentais e drivers detectados após a utilização de bancadas virtuais como mediação hipercultural no processo de construção de conhecimentos relacionados ao comportamento dual da matéria e da radiação eletromagnética.

As atividades propostas por esta pesquisa foram realizadas com um grupo de 12 estudantes, cursando o sétimo período do curso de Licenciatura em Física, de um total de oito da grade curricular vigente. O desenvolvimento dessa atividade será apresentado em quatro momentos, sendo eles:

MOMENTO I: No primeiro momento, um pré-teste ${ }^{1}$ foi construído e validado pelos autores e, posteriormente, aplicado aos estudantes, antes que os mesmos tivessem qualquer contato com os softwares que serão utilizados como proposta deste trabalho. Os pré-testes foram resolvidos individualmente pelos estudantes.

MOMENTO II: Nesta fase, foi entregue aos alunos um roteiro de atividades ${ }^{2}$ para conduzir a utilização dos dois softwares propostos. Os simuladores foram empregados segundo a abordagem P.O.E., Predizer-Observar-Explicar, na qual os estudantes são chamados a predizer o comportamento de uma situação-problema ou de um experimento, observar a simulação e, após essas etapas, explicar possíveis diferenças entre suas concepções e o observado (Wu et al., 2001). É durante este estágio, com duração de 6 horas/aula, que acreditamos ocorrer a internalização dos drives intrínsecos às representações computacionais. O software A - Interferômetro Virtual de MachZehnder (IMZ) (Figura 1-I) permite ao usuário observar o fenômeno de interferência produzido por um feixe de luze por fótons individuais. O software $B$ - Arranjo Experimental da Fenda Dupla de Young (AEDF) (Figura 1-II) permite observar o comportamento de objetos clássicos e quânticos ao passarem por fendas estreitas e muito próximas. 
Figura 1- (I) Interferômetro Virtual de Mach-Zehnder (II) Arranjo experimental virtual da Fenda Dupla de Young.

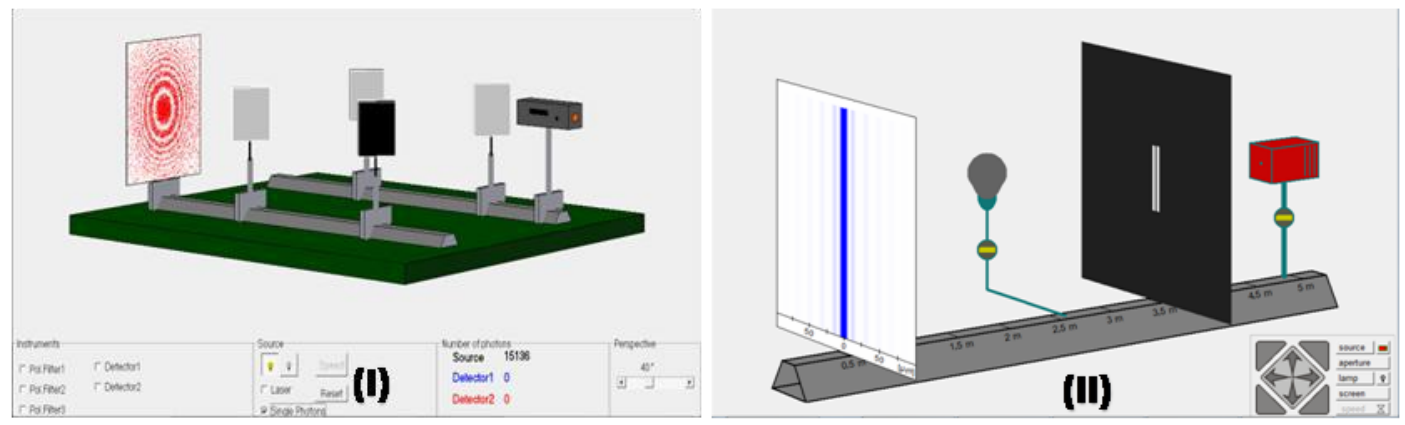

Fonte: Extraídos dos softwares.

MOMENTO III: Na semana seguinte ao término das atividades utilizando os softwares, foi aplicado aos alunos o pós-teste ${ }^{3}$, contendo questões abertas e fechadas referentes ao mesmo conteúdo abordado no pré-teste, o comportamento dual da matéria e da radiação eletromagnética.

MOMENTO IV: Este momento consiste em entrevistas realizadas individualmente com os alunos, tendo como pauta as questões e as respostas do pós-teste. As entrevistas foram conduzidas conforme o protocolo "Report Aloud", sendo uma adaptação da técnica "Think Aloud" (Van-Someren et al., 1994). Essa técnica utiliza um método de coleta de dados em que, basicamente, o entrevistador e o entrevistado mantêm constante diálogo a respeito do que o entrevistado está pensando durante a execução de uma tarefa. As entrevistas foram gravadas e transcritas, tal como os áudios produzidos pelos alunos (sic), para a devida análise.

\section{Resultados e Análise}

Após a realização de todas as entrevistas, a etapa seguinte passa a ser a análise dos dados. Para tanto, todas as entrevistas foram transcritas e os demais instrumentos de coleta de dados foram separados. A linguagem verbal, presente nas transcrições das entrevistas, e a linguagem escrita, presente nos pré e pós-testes, foram apreciadas com base na análise textual discursiva de Moraes e Galiazzi (2007). Por sua vez, a linguagem não verbal, caracterizada pelos gestos descritivos realizados pelos estudantes durante as entrevistas, e principal ferramenta de identificação de drivers adotada, foi analisada a partir da adaptação da metodologia oferecida pela linha de trabalho de Monaghan e Clement (1999).

Neste trabalho, opta-se pela apresentação do perfil epistemológico de dois alunos. Como a proposta de construção de tais perfis está fundamentada e diretamente vinculada às interpretações privadas, imagens mentais e drivers a respeito da dualidade onda-partícula, os perfis epistemológicos apresentados são de alunos que já tiveram suas interpretações analisadas e apresentadas por Trevisan e Andrade Neto (2014), sendo esta abordagem epistemológica atual, um processo investigativo posterior à pesquisa apresentada pelos autores.

\subsection{Esboçando o Perfil Epistemológico do aluno A1}

Por meio dos dados coletados dos questionários e entrevista, indicadores que são utilizados para esboçar o perfil epistemológico acerca da noção da dualidade de A1, 
emergem à medida que a interpretação do aluno diante dos fenômenos observados, bem como as suas representações e imagens mentais vão sendo percebidas e examinadas.

Seguindo o exemplo de Bachelard (1991), será primeiramente apresentado o diagrama esboçando as tendências filosóficas do indivíduo, configurando seu perfil epistemológico. Em seguida, apresentam-se as justificativas para determinada construção espectral filosófica.

Figura 2 - Perfil epistemológico do A1 acerca da noção pessoal da dualidade.

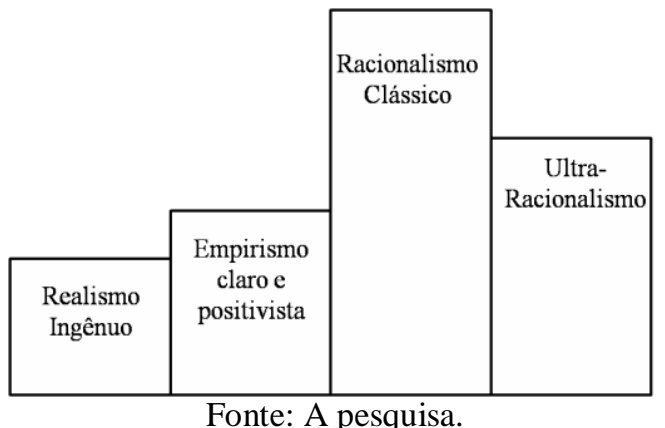

No eixo das abcissas, são indicadas as filosofias sucessivas, e no eixo das ordenadas, um valor que corresponde à frequência efetiva com que tal filosofia é expressada por A1, a respeito de sua noção da dualidade. Dentre as quatro principais filosofias apresentadas no gráfico, a visão racionalista clássica destaca-se, aparecendo com maior influência na construção da noção da dualidade. Esse destaque deve -se à compreensão apresentada por A1 diante dos fenômenos observados nas bancadas virtuais, resultante de sua imaginação para a mudança do comportamento dos objetos quânticos, de sua visão corpuscular para a ondulatória. Portanto, a teoria utilizada ao descrever a origem dos pontos de "máximo e mínimo" (A1) converge à postura racionalista clássica.

Já a postura filosófica menos presente é a representada pelo realismo ingênuo, sendo esse o pensamento mais primitivo do indivíduo. É manifestado, por exemplo, nas representações do aluno para os objetos quânticos, como a imagem de "uma esfera brilhante" para o fóton, associando, possivelmente, esse brilho da esfera ao produzido pelo aquecimento dos filamentos ou gases das lâmpadas.

As filosofias que representam o empirismo claro e positivista e o ultraracionalismo permeiam entre si e revelam-se com intensidades próximas nesse perfil. A postura empirista e positivista aparece com a necessidade do aluno em experimentar e observar (realizar uma "medição") em determinadas situações, para que, então, possa averiguar e utilizar determinado comportamento da luz (e elétrons, por exemplo) para interpretar e explicar certos desdobramentos dos fenômenos. Portanto, como consequência dessa conduta, o A1 afirma não saber a natureza da luz e objetos quânticos sem antes analisá-los em situações específicas, dessa forma sua resposta estará vinculada a determinadas condições de "contorno".

Neste seguimento, o aluno mostra consciência a respeito da dualidade apresentada pelos objetos quânticos, afirmando interpretá-los ora como onda (dentro do setup experimental) e ora como partícula (isolado). Este grau de pensamento encaixa-se na postura ultra-racionalista - mesmo que de forma incipiente - tendo em vista os obstáculos epistemológicos superados em sua fase de formação, sendo essa uma interpretação oriunda da "Física Moderna e Contemporânea". Essa filosofia não recebe maior representatividade pelo fato, por exemplo, de o aluno não prever em seu guia de atividade o padrão formado no anteparo do IMZ quando a fonte opera em regime 
monofotônico, sendo que em seu pós-teste a resposta para o mesmo questionamento condiz com o resultado apresentado pelo software. Possivelmente esta evolução na concepção do fenômeno é consequência da manipulação das ferramentas hiperculturais propostas.

O perfil epistemológico de A1 condiz com a interpretação privada (ainda não consolidada) Dualista Positivista. Os drivers, imagens mentais e as representações utilizadas e detectadas pelo aluno permitiram o esboço filosófico considerado primário no indivíduo, formando uma conduta que o guiará em determinadas interpretações para fenômenos e conceitos específicos. É importante ressaltar que o perfil epistemológico "[...] deve sempre referir-se a um conceito designado, de ele apenas ser válido para um espírito particular que se examina num estádio particular da sua cultura" (Bachelard 1991, p. 25).

\subsection{Esboçando o Perfil Epistemológico do aluno A2}

Após a investigação da interpretação, drivers e imagens mentais utilizadas pelo estudante para resolver e entender os fenômenos visualizados nas ferramentas hiperculturais em forma de bancadas virtuais e nos questionários pré e pós-teste, apresenta-se a seguir o esboço do perfil epistemológico acerca da noção da dualidade do A2.

Figura 3 - Perfil epistemológico do A2 acerca da noção pessoal da dualidade.

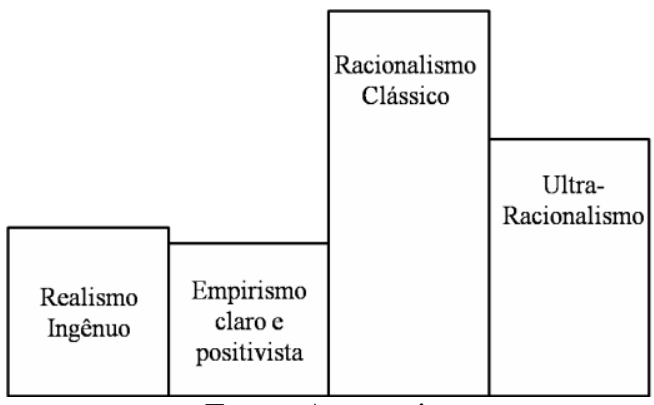

Fonte: A pesquisa.

O gráfico construído mostra que o racionalismo clássico é a filosofia com maior representatividade na formação da noção da dualidade do A2, acompanhada, em segundo plano, pela atitude ultra-racionalista, que também ganha destaque na construção da compreensão sobre o tema.

O racionalismo clássico desponta na interpretação dualista realista exibida pelo aluno ao longo das atividades. Alegando maior facilidade em explicar o comportamento ondulatório do elétron e do fóton - que na concepção do aluno são considerados partículas -, A2 utiliza a teoria ondulatória clássica ao esclarecer os padrões de interferência vistos no anteparo. Essa postura conecta-se com o racionalismo clássico presente em seu perfil, filosofia reforçada com a lógica quântica expressada pelo aluno ao dar a primeira explicação valendo-se da falta de informação do caminho (no IMZ) e da Fenda (no AEDF) percorridos pelo fóton, tal raciocínio apenas é reproduzido, mas não compreendido. Por esse motivo, A2 pondera ter maior compreensão imaginando uma onda associada aos objetos quânticos. O aluno afirma ser mais "fácil" explicar os fenômenos considerando o elétron e fóton com comportamento ondulatório, no entanto, garante que esse raciocínio é incorreto. Essa é uma evidência da falta de conhecimento das diversas interpretações da MQ, em especial a ondulatória de Schrödinger.

O ultra-racionalismo, ainda que incipiente em sua atitude, mostra a consciência do A2 para a dualidade dos objetos quânticos. O aluno afirma ser mais confortável 
explicar a dualidade do fóton e do elétron nos experimentos do IMZ e da Dupla fenda, com ambos os objetos quânticos possuindo ondas a eles associadas, sendo que, pela falta de conhecimento das principais interpretações da MQ, o aluno considera esse raciocínio como sendo incorreto "cientificamente". O empirismo claro e positivista e o realismo ingênuo contribuem em menor frequência para a noção do aluno.

As representações e imagens mentais, reproduzindo sua concepção em relação ao fóton e elétron, podem caracterizar sua parcela que define o realismo ingênuo como terceira filosofia com maior representatividade no esboço do perfil epistemológico de A2. Um exemplo é o da imagem mental do estudante para a onda associada aos objetos quânticos, na qual o elétron ou o fóton possuem um rabinho representando a sua componente ondulatória. Essa representação é evidenciada como incorreta pelo aluno que utiliza como referência a fala de seu professor em sala de aula. Portanto, o aluno não se permite utilizar explicitamente tal imagem mental. Desta forma, verifica -se que as imagens mentais do A2 estão repletas de imagens do realismo ingênuo.

Assim, ainda que de maneira superficial, construiu-se o esboço do perfil epistemológico do A2, determinando possíveis evidências de como as diversas filosofias relacionam-se com o conceito da dualidade.

\section{Considerações Finais}

A Teoria Quântica é considerada uma teoria robusta e de sucesso. No entanto, é uma característica notável dessa teoria o fato de ela ser interpretada de diferentes maneiras, sendo que cada uma dessas interpretações é internamente consistente e, de modo geral, congruente com os experimentos quânticos. Assim sendo, perguntas como "do que se trata o formalismo quântico?" e "a que se referem seus símbolos e equações?" possuem respostas distintas, pois dependem diretamente do ponto de vista (interpretação) tomado. Essa possibilidade de adoção de diferentes interpretações na MQ talvez possa ser considerada o cerne para o seu ensino.

Desta forma, acreditamos na importância da investigação a respeito das filosofias que compõem o espírito do sujeito na construção das interpretações acerca da dualidade, com a intenção de mapear as dificuldades de compreensão dos problemas fundamentais da MQ. Nesta proposta, o perfil epistemológico dos alunos pode ser traçado (ainda que de maneira incipiente) de acordo com as interpretações privadas, inferidas a partir dos drivers conceituais, manifestados durante as entrevistas, onde foram comparados com as quatro principais interpretações da MQ. Consideramos que a contribuição principal dessa pesquisa seja justamente a interligação entre as imagens mentais e drivers, averiguados ao longo das atividades desempenhadas com os alunos, e o perfil epistemológico dos estudantes a respeito do comportamento da radiação eletromagnética e de objetos quânticos.

Após a identificação da importância do ensino da MQ, mantendo a preocupação na abordagem conceitual, não apenas nos processos de cálculos matemáticos, e também a crescente aparição das TIC, voltadas aos processos educativos, projetamos dar sequência à investigação do tema, pesquisando instrumentos que contribuam no levantamento das filosofias utilizadas pelos estudantes diante de situações problemas que envolvam temas fundamentais da MQ. Pretendemos também investigar o desenvolvimento e a utilização de ferramentas hiperculturais em forma de simulações computacionais que preencham a lacuna representacional do comportamento do mundo microscópico em experimentos fundamentais da TQ, permitindo ao aluno observar as representações e drivers vinculados às principais interpretações MQ. 


\section{Notas de Texto}

${ }^{1}$ Disponibilizado em: http://migre.me/mOBbm

${ }^{2}$ Disponibilizado em: http://migre.me/mODjQ

${ }^{3}$ Disponibilizado em: http://migre.me/mODwd

\section{Referências Bibliográficas}

BACHELARD, G. A Filosofia do Não - Filosofia do Novo Espírito Científico. 5.ed. Lisboa: Presença, 1991.

GRECA, I. M.; MOREIRA, M. A.; HERSCOVITZ, V. E. Uma proposta para o ensino de mecânica quântica. Revista Brasileira de Ensino de Física, São Paulo, v. 33, n. 4, p. 444-457, dez. 2001.

MONAGHAN, J. M.; CLEMENT, J. J. Use of a computer simulation to develop mental simulations for understanding relative motion concepts. International Journal of Science Education, v. 21, n. 9, p. 921 - 944, 1999.

MONTENEGRO, R.L.; PESSOA JR., O. Interpretações da Teoria Quântica e as Concepções dos Alunos do Curso de Física. Investigações sobre Ensino de Ciências, v.7, n.2, 2002.

MORAES, R; GALIAZZI, M. C..Análise Textual Discursiva. Ijuí: Editora Unijuí, 2007.

OSTERMANN, F.; PRADO, S. D. Interpretações da mecânica quântica em um interferômetro virtual de Mach-Zehnder. Revista Brasileira de Ensino de Física, v. 27, n. 2, p. 193-203, 2005.

PESSOA JR, O. Conceitos de física quântica. São Paulo: Livraria da Física,v. 2, 2008. PINTO, A.C., ZANETIC, J. É Possível Levar a Física Quântica para o Ensino Médio? Caderno Catarinense de Ensino de Física, Florianópolis, v. 16, n. 1, p. 7-34, abr. 1999.

ROCHA, J. F. M. Origem e Evolução do Eletromagnetismo. In: ROCHA, José Fernando Moura (org). Origens e Evolução das Idéias da Física. Salvador: EDUFBA, 2002.

SCHUCK, A. F.; SERRANO, A. Um exemplo do uso de experimentos virtuais objetivando a introdução de conceitos de mecânica quântica na disciplina de estrutura da matéria. In: IX Encontro Nacional de Pesquisa em Ensino de Física. Anais.. p.110, 2004.

SOUZA, B. C.. A Teoria da Mediação Cognitiva: os impactos cognitivos da hipercultura e da mediação digital. 2004. 282 f. Tese (Doutorado) - Curso de Psicologia, Universidade Federal de Pernambuco, Recife, 2004.

SOUZA, B. C.; SILVA, A. S. ; SILVA, A. M. ; ROAZZI, A. ; CARRILHO, S. L. S. . Putting the Cognitive Mediation Networks Theory to the test: Evaluation of a framework for understanding the digital age. Computers in Human Behavior, v. 007, p. 10.1016, 2012.

TREVISAN, R.; ANDRADE NETO, A. S. . A Utilização de Ferramentas Hiperculturais no Ensino de Mecânica Quântica: Investigação do Aprendizado de Representações, Drivers e Conceitos Quânticos. RENOTE - Revista Novas Tecnologias na Educação, v. 12, p. 1, 2014.

VAN-SOMEREN, M. W.; BARNARD, Y. F.; SANDBERG, J. A. C. The Think Aloud Method: a practical guide to modeling cognitive processes. London, 1994.

WU, H.-K.; KRAJCIK, J.S.; SOLOWAY, E. Promoting Understanding of Chemical Representations: Students' Use of a Visualization Tool in the Classroom. Journal of Research in Science Teaching, v.38, n.7, p. 821-842, 2001. 